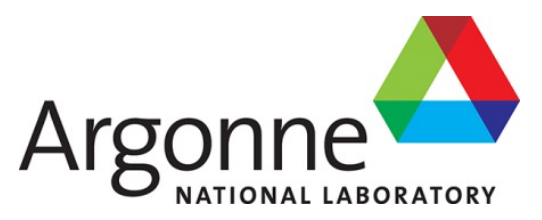

\title{
A Scalable Mixed-Integer Decomposition Method for Security-Constrained Optimal Power Flow with Complementarity Constraints
}

Final Scientific/Technical Report

Mathematics and Computer Science Division 


\begin{abstract}
About Argonne National Laboratory
Argonne is a U.S. Department of Energy laboratory managed by UChicago Argonne, LLC under contract DE-AC02-06CH11357. The Laboratory's main facility is outside Chicago, at 9700 South Cass Avenue, Lemont, Illinois 60439. For information about Argonne and its pioneering science and technology programs, see www.anl.gov.
\end{abstract}

\title{
DOCUMENT AVAILABILITY
}

Online Access: U.S. Department of Energy (DOE) reports produced after 1991 and a growing number of pre-1991 documents are available free at OSTI.GOV (http://www.osti.gov/), a service of the US Dept. of Energy's Office of Scientific and Technical Information.

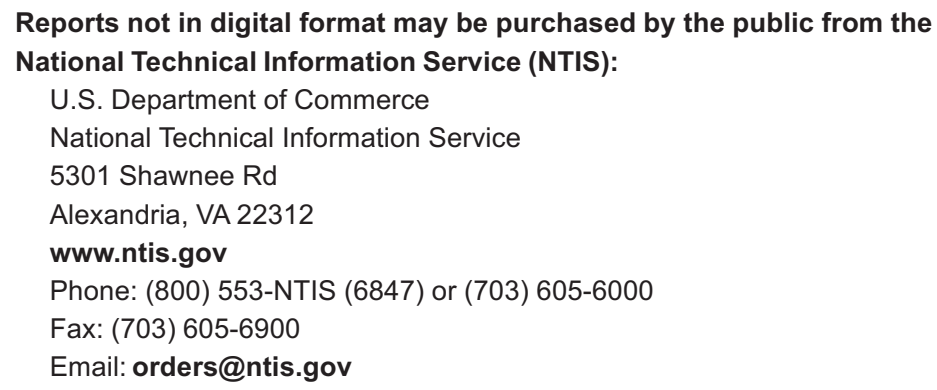

Reports not in digital format are available to DOE and DOE contractors from the Office of Scientific and Technical Information (OSTI):

U.S. Department of Energy

Office of Scientific and Technical Information

P.O. Box 62

Oak Ridge, TN 37831-0062

www.osti.gov

Phone: (865) 576-8401

Fax: (865) 576-5728

Email: reports@osti.gov

\section{Disclaimer}

This report was prepared as an account of work sponsored by an agency of the United States Government. Neither the United States Government nor any agency thereof, nor UChicago Argonne, LLC, nor any of their employees or officers, makes any warranty, express or implied, or assumes any legal liability or responsibility for the accuracy, completeness, or usefulness of any information, apparatus, product, or process disclosed, or represents that its use would not infringe privately owned rights. Reference herein to any specific commercial product, process, or service by trade name, trademark, manufacturer, or otherwise, does not necessarily constitute or imply its endorsement, recommendation, or favoring by the United States Government or any agency thereof. The views and opinions of document authors expressed herein do not necessarily state or reflect those of the United States Government or any agency thereof, Argonne National Laboratory, or UChicago Argonne, LLC. 


\section{A Scalable Mixed-Integer Decomposition Method for Security- Constrained Optimal Power Flow with Complementarity Constraints}

Final Scientific/Technical Report

prepared by

Kibaek Kim, Youngdae Kim, Daniel A. Maldonado, Michel Schanen, and Victor M. Zavala Mathematics and Computer Science, Argonne National Laboratory

Nai-Yuan Chiang

United Technologies Research Center

November 24, 2021 
Final Scientific/Technical Report

Argonne National Laboratory

A Scalable Mixed-Integer Decomposition Method for Security-Constrained Optimal Power Flow with Complementarity Constraints

1952-1556

\begin{tabular}{|c|c|}
\hline Award: & DE-AR0001088 \\
\hline Sponsoring Agency: & USDOE, Advanced Research Project Agency - Energy (ARPA-E) \\
\hline Lead Recipient: & \begin{tabular}{c} 
Argonne National Laboratory \\
\hline Project Team Members:
\end{tabular} \\
\hline Project Title: & $\begin{array}{r}\text { A Scalable Mixed-Integer Decomposition Method for Security- } \\
\text { Maldonado, Michel Schanen, Victor M. Zavala } \\
\text { Constrained Optimal Power Flow with Complementarity } \\
\text { Constraints }\end{array}$ \\
\hline Program Director: & Dr. Kory Hedman \\
\hline Principal Investigator: & Dr. Kibaek Kim \\
\hline Contract Administrator: & Dino Marconi \\
\hline Date of Report: & $11 / 24 / 2021$ \\
\hline Reporting Period: & $02 / 01 / 2019-05 / 29 / 2020$ \\
\hline
\end{tabular}

The information, data, or work presented herein was funded in part by the Advanced Research Projects Agency-Energy (ARPA-E), U.S. Department of Energy, under Award Number DEAR0001088. The views and opinions of authors expressed herein do not necessarily state or reflect those of the United States Government or any agency thereof.

This report contains no protected data. 
Table of Contents

\section{Table of Figures/Tables}

Table 1. Key Milestones and Deliverables.

$\mathrm{x}$

\section{Public Executive Summary}

This project aimed to develop a scalable algorithm for security-constrained optimal power flow (SCOPF) under contingency scenarios. In particular, the SCOPF problem targeted in the GO Competition is challenging because of the nonconvexity, its nonsmoothness, and the problem size, which increases with the number of contingency events. Complementarity constraints imposed in post-contingency variables are particularly challenging because they lead to a violation of constraint qualifications at any feasible point.

For the competition we developed a novel mixed-integer decomposition approach that exploits the two-stage structure of the problem and that directly handles the complementarity constraints. The key idea of our solution approach is based on the primal and dual representations of the original SCOPF. The dual approach is based on the Lagrangian relaxation of SCOPF with respect to the constraints that couple the pre-contingency and post-contingency states. The dual approach results in many smaller subproblems that are solved in parallel on the GO Competition's computing cluster. The primal representation is used to find feasible solutions by the parallel interior-point solver PIPS-NLP. All the models and algorithms have been written in Julia except for PIPS-NLP, an Argonne's existing software library written in C++.

The developed solution method is effective for finding high-quality solutions of the SCOPF problem in parallel. However, we determined that the method was not guaranteed to find a feasible solution within a short time limit, which was an important criterion for the competition evaluation. We identified as a promising future direction the development of heuristic algorithms with the proposed method, which can address the main drawback of the method. We also emphasize that the developed solution method is generic and applicable to other problem classes beyond SCOPF. In fact, the key idea of this project has been adapted to the DOE Exascale Computing Project in collaboration with other national laboratories.

\section{Acknowledgments}

This material is based upon work supported by the U.S. Department of Energy, Advanced Research Projects Agency-Energy, under contract DE-AC02-06CH11357. We gratefully acknowledge the computing resources provided on Bebop, a high-performance computing cluster operated by the Laboratory Computing Resource Center at Argonne National Laboratory. 


\section{Accomplishments and Objectives}

This award allowed Argonne National Laboratory to demonstrate a number of key objectives. The focus of the project was on (i) developing a set of optimization formulations and algorithms for solving security-constrained optimal power flow under contingency scenarios; (ii) testing the solution approaches to the GO Competition software platform; and (iii) reporting the performance results to the leaderboards.

The actual performance against the stated milestones is summarized in the following table.

Table 1. Key milestones and deliverables

\begin{tabular}{|l|l|l|}
\hline Stage & Tasks & \multicolumn{1}{c|}{ Task Description } \\
\hline 1 & 1 & $\begin{array}{l}\text { Register for the GO Competition on the competition website } \\
\text { Actual Performance: This has been completed as planned. }\end{array}$ \\
\hline 2 & 2 & $\begin{array}{l}\text { Test our software approach at least once by month 3 of the GO Competition } \\
\text { (by using the GO Competition software platform); results must be displayed } \\
\text { on the leaderboards. } \\
\text { Actual Performance: This has been completed as planned. }\end{array}$ \\
\hline 3 & 3 & $\begin{array}{l}\text { Participate in the Trial 1 event at month 6; results must be displayed on the } \\
\text { leaderboards. } \\
\text { Actual Performance: This has been completed as planned. }\end{array}$ \\
\hline 4 & 4 & $\begin{array}{l}\text { Participate in the Trial 2 event at month 9; results must be displayed on the } \\
\text { leaderboards. } \\
\text { Actual Performance: This has been completed as planned. }\end{array}$ \\
\hline 5 & 5 & $\begin{array}{l}\text { Participate in the Challenge 1 Final Event; results must be displayed on the } \\
\text { leaderboards. } \\
\text { Actual Performance: This has been completed as planned. }\end{array}$ \\
\hline
\end{tabular}

\section{Project Activities}

The project has focused on developing a set of formulation techniques (i.e., reformulation, relaxation) and a decomposition method for solving the SCOPF problem posed by the GO Competition. The major approach/hypothesis weas that the developed method can use a sufficiently large number of computing processes and sufficiently large memory for parallel computing. We spent significant effort on resolving the issues with limited resources (i.e., processes, memory, competition time limit). Faced with these major challenges for the project, we successfully investigated a number of heuristic and approximation algorithms.

We have implemented the algorithms in the Julia programming language, which allowed us to rapidly prototype multiple algorithms and their variations. In particular, we have implemented a decomposition algorithm that separates the preventive control and reactive controls (i.e., controls after contingency) of SCOPF. To accelerate the solutions, we have implemented the 
second-order cone relaxation, quadratic-constraint relaxation, and linear relaxation, each of which has been used in a hierarchical way. The complementarity constraints required in the reactive control stage have been modeled in two ways: (1) by introducing binary variables and (2) by approximating with nonlinear inequalities.

The major weakness learned from this project is that Julia code can be extremely slow when starting from scratch, which can only worsen when creating large-scale optimization problems. In most research projects, this may not be an issue. But the slow startup was serious for the GO competition. The other issue we struggled with was problems with the Julia package versions, which ran on our machines but not on the competition machine, over which our team did not have control. We greatly appreciate the competition support staff who helped us with these struggles. Unfortunately, we had to spend most of our time dealing with this issue.

Nevertheless, we emphasize that the ideas and experience learned from this project have been successfully applied to our Exascale Computing Project and in multiple software packages and papers targeted on GPUs [1-4]. In particular, the decomposition method proposed for this project has been extended for solving large-scale multiperiod ACOPF on multiple GPUs on the Summit supercomputer at Oak Ridge National Laboratory.

\section{References}

1. Schanen, Michel, Daniel Adrian Maldonado, François Pacaud, Alexis Montoison, Mihai Anitescu, Kibaek Kim, Youngdae Kim, Vishwas Rao, and Anirudh Subramanyam. "Julia as a portable high-level language for numerical solvers of power flow equations on GPU architectures." Les Cahiers du GERAD ISSN 711 (2020): 2440.

2. Zhang, Weiqi, Kibaek Kim, and Victor M. Zavala. "On the tightness and scalability of the Lagrangian dual bound for the alternating current optimal power flow problem." arXiv preprint arXiv:2104.03788 (2021).

3. Kim, Youngdae, François Pacaud, Kibaek Kim, and Mihai Anitescu. "Leveraging GPU batching for scalable nonlinear programming through massive Lagrangian decomposition." arXiv preprint arXiv:2106.14995 (2021).

4. Kim, Youngdae, and Kibaek Kim. "Accelerated computation and tracking of AC optimal power flow solutions using GPUs." arXiv preprint arXiv:2110.06879 (2021). 


\section{Argonne}

Mathematics and Computer Science Division

Argonne National Laboratory

9700 South Cass Avenue, Bldg. \#240

Lemont, IL 60439

www.anl.gov 